\title{
Online Birth Plans and Anticipatory Guidance: A Critical Review Using Web Analytics and Crowdsourcing
}

\author{
Christina D. Yarrington, MD \\ Kari Radoff, CNM
}

Chloe A. Zera, MD

\begin{abstract}
We assessed the content of online birth plans using Web analytics and crowdsourcing to identify the most commonly accessed online birth plans. We analyzed the first 14 websites of 6 different searches across time. We repeated this search using 200 individuals recruited by a commercial crowdsourcing service. We identified the 12 URLs that appeared most frequently from all searches then categorized the content by relevance to stages of labor or newborn care. Twenty-five different URLs with customizable birth plans were identified by clinical and crowdsourced searches, with considerable overlap. Among the most commonly identified birth plans, we found prompts for outdated practices in a minority $(18 \%-37 \%)$. The majority of online birth plans can be positive tools for shared decision making.
\end{abstract}

The Journal of Perinatal Education, 27(1), http://dx.doi.org/10.1891/1058-1243.27.1.32

Keywords: birth plans, internet, prenatal education

\section{INTRODUCTION}

A birth plan is a document created by an expectant mother, or parents, that both communicates preferences about the birth experience to her attendants and also facilitates her own values clarification (Mei, Afshar, Gregory, Kilpatrick, \& Esakoff, 2016). Birth plans have been suggested as a modality to improve patient satisfaction with the birth experience by addressing expectations, involvement in decision making, and the quality of the caregiverwoman relationship (Lothian, 2006). Women may receive guidance on writing a birth plan from childbirth education classes or lay literature, including interactive websites (Bailey, Crane, \& Nugent, 2008). Recent research by Google Consumer Surveys demonstrates that new and expecting parents do twice as many searches as nonparents, with health as the top search item (Rost, Johnsmeyer, \& Mooney, 2014). The 2013 National Institutes of Health-funded report Listening to Mothers III-a survey of pregnant women across the United States-found that the vast majority of respondents used the internet 
as a source of information about pregnancy: $82 \%$ of respondents used a laptop or desktop with internet access and $64 \%$ used a smartphone with internet access during a typical week of pregnancy, with utilization similar across race and ethnicity. A recent study found that $39 \%$ of expectant mothers used the internet as a source for information specifically for their birth plan (Pennell, Salo-Coombs, Herring, Spielman, \& Fecho, 2011). Despite the ubiquity of an internet search for information, the content about labor and birth may not always be reliable (Lothian, 2006; Kaufman, 2007). Knowledge of patientidentified resources about the birth experience can optimize prenatal education and counseling by the clinical team. However, clinicians attempting their own Web search for birth plans will find over 30 million hits. Little is known about which sites childbearing women most frequently access, what the birth plans routinely contain and how the content in these sites may guide preferences.

In this study, we therefore sought to identify what expectant and first-time mothers commonly find when they search online for birth plans as well as to categorize the type and relevance of this information. Our search was performed and refined using techniques of Web analytics, processes that study the access and use of information on the internet. We validated our search with results obtained from crowdsourcing, a method of obtaining content by soliciting contributions from a large group of people.

\section{MATERIALS AND METHODS}

The Institutional Review Board at Brigham and Women's Hospital Boston, MA reviewed the project design and determined that it met the criteria for exemption 45 CFR 46.101(b). We searched six iterations of the search terms for a birth plan template. The first was intended to be the simplest with inclusion of the key phrases "birth plan," "free," and "template." The next five were selected based on the suggestions from the Google search engine that included options for birth plan templates. The Google search engine was then used to search for following groups of words without Boolean Operators: "birth plan template free," "sample birth plan template," "simple birth plan template," "birth plan template pdf," "good birth plan template," and "printable birth plan template." Based on data from Web analytics that click through rates in Google searches nadir at hit number 14 with only $1.1 \%$ of browsers clicking on the link (Goodwin, 2011), the
Birth plans have been suggested as a modality to improve patient satisfaction with the birth experience by addressing expectations, involvement in decision-making, and the quality of the caregiverwoman relationship.

first two pages of results retrieved were listed and the first 14 were accessed. Only websites that linked to birth plans that could be customized and printed were included for analysis. We excluded websites that gave examples of other families' birth plans or that described the principles of a birth plan.

To validate our search results, we used Crowdflower, a crowdsourcing service that coordinates participants and employers for time limited Web-based projects, to recruit participants from across the country. Through Crowdflower, we recruited 200 individuals to provide the URLs for the first two online customizable birth plans they found. Workers participated from 42 different nations in North and South America, Asia, and Europe. Crowdflower does not provide detailed worker demographics. Participants were compensated $\$ 0.20$ for their contribution, the suggested rate for a task taking less than 2 minutes. These results were reviewed and each website accessed to ensure it linked to a customizable birth plan.

Prompts or preferences listed in the birth plan template were then categorized according to elements related to the first, second, and third stage of labor as well as newborn care. Preferences for circumcision were not included in the analysis as this procedure requires explicit written consent. Frequency of a given element was described as a percentage of the top 10 plans accessed by a research investigator and percentage of the top 10 plans retrieved by crowdsourcing, as well as the top 3 plans of all combined results.

We described the websites identified by the research investigator with a mean rank and a weighted mean rank. The weighted mean rank included the times a given site appeared on multiple searches and was calculated by dividing the mean rank by the number of times a site appeared in the top 14 links of the 12 searches. Descriptive

\section{Little is known about which sites childbearing women most}

frequently access, what the birth plans routinely contain and how

the content in these sites may guide preferences. 
statistics were used to describe frequency of elements in birth plans and compared with student's $t$ test. Frequency of identification of a given URL was also described with percentage in the analysis of crowdsourced data.

\section{RESULTS}

A total of 12 searches executed and reviewed as described above by a research investigator yielded a total of 25 different websites that allowed customization of birth plans. The top 10 sites presented are those with a weighted rank less than two that appeared at least five times among rank lists.

Crowdflower returned a total of 400 URLs done by 200 different workers. After review and elimination of sites that did not link to the requested information, the crowdsourced data resulted in 281 URLs (70.2\% yield) with customizable birth plans. The top 10 sites of the Crowdsourced cohort were identified according to the number of times a crowdsourcing worker identified that URL. There was substantial overlap between the URLs identified by a research investigator in Boston and the URLs identified by an international group of workers. The top 10 URLs identified by crowdsourcing included eight of the top 10 identified by the research investigator. The top three sites were the same for the research investigator and the crowdsourced cohort.

One of the top websites from both searches was a site that linked to 10 different sites with customizable birth plans, 5e of which were already listed independently in many of the search results. The frequency of different birth plan elements for this website was determined by taking an average of all 10 plans.

Merging the top 10 searches of the clinician and the crowdsourced data resulted in a list of 12 URLs displayed in Table 1. Table 2 demonstrates the frequency of each birth plan element from the combined top 12 sites as well as in the common top 3 sites categorized by stage of labor or newborn care. When the top 10 crowdsourced birth plans were compared to the top 10 birth plans identified by the clinical researcher there was no difference in the prevalence of any of the elements listed.

\section{DISCUSSION}

While there is a common apprehension about documents obtained through the internet, our research found the most commonly accessed online customizable birth plans included reasonable prompts for preferences and values clarification and outdated practices were referenced in only a minority of plans. Our data suggest that most of the content in online birth plans supports a positive birth experience with categories of preferences that can be met by a thoughtful perinatal team. The most common elements identified (present in $75 \%-100 \%$ of birth plans) can easily be addressed in early visits, in the context of labor and birth unit or birthing center tours, or in written handouts in planning a woman's birth. Overall, prompts that suggested practices no longer customary in the United States such as routine enema and shaving for vaginal birth were rare (36\% and $18 \%$, respectively).

Over half included a prompt regarding preferences for immediate administration of intramuscular vitamin $\mathrm{K}$, recommended by the American Academy of Pediatrics (AAP) to reduce the risk of vitamin K-dependent hemorrhage (AAP, 1992, $2009,2012)$. Several of the sites included the preference for oral rather than intramuscular vitamin $\mathrm{K}$. The AAP recommendation for intramuscular injection only is also supported by the Canadian

TABLE 1

Top 12 URLs of Modifiable Birth Plans Identified by Clinical and Crowdsourced Searches

\begin{tabular}{ll}
\hline Rank & URL \\
\hline 1 & http://thebump.com/a/tool-birth-plan/ \\
2 & http://babycentre.co.uk/i/Antenatalclasses/birth-plan-notes.pdf \\
3 & http://blog.earthmamaangelbaby.com/birth-plan/ \\
4 & http://justmommies.com/quizzes/birthplan.php \\
5 & http://childbirth.org/interactive/ibirthplan.html \\
6 & http://couponkarma.com/10-free-printable-pregnancy-birth-plans-hospital-bag-checklists-for-2013/ \\
7 & http://www.bellybelly.com.au/birth/birth-plan-can-you-plan-birth/ \\
8 & http://www.bubhub.com.au/hubbub-blog/birth-plan-template/ \\
9 & http://nhs.uk/conditions/pregnancy-and-baby/pages/birth-plan.aspx \\
10 & http://birthwithoutfearblog.com/2012/12/04/birth-and-post-birth-plans-drafts-to-download-modify-and-use/ \\
11 & http://pregnancyandbaby.com/calendars/articles/937331/birth-plan-creator/ \\
12 & http://www.sheknows.com/parenting/articles/818481/How-to-write-your-ideal-birth-plan \\
\hline
\end{tabular}


TABLE 2

Prevalence of References and Indications in Top 12 Online Customizable Birth Plans

\begin{tabular}{|c|c|c|c|c|}
\hline Stage of labor & $75 \%-100 \%$ of birth plans & $50 \%-75 \%$ of birth plans & $25 \%-50 \%$ of birth plans & $15 \%-25 \%$ of birth plans \\
\hline First & $\begin{array}{l}\text { - Pain relief } \\
\text { - People present } \\
\text { - Position for labor } \\
\text { - Fetal monitoring } \\
\text { - AROM }\end{array}$ & $\begin{array}{l}\text { - } \\
\text { Augmentation } \\
\text { preferences } \\
\text { - } \text { Intravenous } \\
\text { preferences }\end{array}$ & $\begin{array}{l}\text { - } \text { Ambiance in room } \\
\text { - } \text { Desire to eat-drink } \\
\text { ad lib } \\
\text { - Enema } \\
\text { - } \text { Min SVEs }\end{array}$ & $\begin{array}{l}\text { - Shave } \\
\text { - Foley } \\
\text { - Single provider }\end{array}$ \\
\hline Second & - Episiotomy & - Position for birthing & & \\
\hline Third & $\begin{array}{l}\text { - Umbilical cord } \\
\text { management }\end{array}$ & - Placental management & & $\begin{array}{l}\text { - Avoid vacuum } \\
\text { - Avoid forceps }\end{array}$ \\
\hline Neonatal care & - Breastfeeding & $\begin{array}{l}\text { - Neonatal exam location } \\
\text { - No vitamin K } \\
\text { - No pacifier }\end{array}$ & $\begin{array}{l}\text { - No formula } \\
\text { - No erythromycin }\end{array}$ & - Delay/defer PKU testing \\
\hline
\end{tabular}

Note. $\mathrm{AROM}=$ artificial rupture of membranes.

Paediatric Society, College of Family Physicians of Canada, and Italian Society of Neonatology. The UK Department of Health allows for either an intramuscular or oral dosing. However, while there is limited data that oral dosing decreases the risk of classic vitamin $\mathrm{K}$-dependent bleeding, it does not appear to reduce the risk of late vitamin $\mathrm{K}$-dependent bleeding, which carries a 50\% risk of intracranial hemorrhage and a 20\% mortality rate (Lippi \& Franchini, 2011). Early education about immediate neonatal care practices may mitigate the influence of otherwise arbitrary checkboxes women will encounter when they start to prepare their birth plan. Whatever their decision, parents' preferences regarding administration of vitamin $\mathrm{K}$ are a valuable part of any birth plan, as a 2013 study of clinician's perspectives on birth plans found that it was one of the most important components to perinatal nurses (Aragon et al., 2013).

Of note the majority of both the clinical search results and the crowdsourced search results included prompts about management of the second stage of labor, specifically, to decline Pitocin to facilitate expulsion of the placenta and uterine tone. Seventyfive percent of the birth plans included an area to indicate preferences for augmentation, and many offered sample statements expressing a desire to avoid augmentation with Pitocin, a preference not unfamiliar to many clinicians. The American College of Obstetrics and Gynecology recommends routine use of Pitocin infusion during the third stage of labor and a recent Cochrane review of the practice demonstrated this practice reduces the rate of postpartum hemorrhage $(\mathrm{NNT}=12)$, severe postpartum hemorrhage, anemia, need for transfusion, and the need for additional uterotonic medications without demonstrated maternal risks (The American College of Obstetricians and Gynecologists, 2006; Westhoff, Cotter, \& Tolosa, 2013). Given the disproportionate benefit of routine use of Pitocin in the third stage, a few words from the clinician to the childbearing woman early in prenatal care may facilitate collaborative decision making.

Cross-sectional work by Aragon et al. (2013) found that when women are questioned about the most important components of a birth plan they include the following as the top four in decreasing order: pain management, comfort measures (including mobility and pushing preferences), postpartum preferences (cord cutting, breastfeeding), and use of interventions (e.g., episiotomy and vacuum or forceps-assisted birth) (Aragon et al., 2013). This is consistent with our findings of the most frequently prompted elements of online birth plans. Their study concluded that the act of creating a birth plan was an educational process for women, reinforcing the importance of early education to address the rationale and indications for intervention when warranted.

Prior studies have shown that women were less satisfied with the birth process when their childbirth did not follow their birth plan compared to women who were able to follow their plan (Mei et al., 2016; Lothian, 2006). Other studies have identified feelings of disappointment and betrayal by their providers when a birth plan was not fulfilled (Carlton, Callister, \& Stoneman, 2005; Too, 1996). Despite the recommendations of these and other studies that flexibility on the part of the birthing woman improves satisfaction and decreases disappointment, only $26 \%$ of our top 12 birth plans included any statement about flexibility. Notably, 
neither of the top two birth plans had any statement about flexibility.

The strengths of our study lie in the methodologies rooted in Web analytics and crowdsourcing. Telescoping the findings of a Web search from the initial report of 33 million to 25 different plans listed repeatedly on various searches by both clinician and lay people enables a realistic snapshot of the information that will be identified by an average birthing woman. The use of crowdsourcing in identifying and reviewing online birth plan templates improves on prior work assessing Web-based obstetric information as it replicates on a large scale the real search a lay person does. Prior work in Web analytics has observed that lay consumers are more likely to use single search terms than health-care professionals (Eysenbach \& Köhler, 2002; Jansen, Spink, \& Saracevic, 2000). We attempted to capture this difference in our method of validation with crowdsourcing by allowing workers to find a customizable birth plans with any search terms they chose. Crowdsourcing also presents possible limitations. There is the potential that the participants, as internet quasi-professionals, could do a more advanced search than a typical childbearing woman. Both the generalizability of crowdsourced findings and the reduced yield of appropriate task completion may be ameliorated by heightening the screening and qualifications required for workers, a service evolving in some crowdsourcing services.

The greatest limitation of our study is in the dynamic growth of the internet. The number of new websites grows daily. Overall, search result order is determined by user click-through rates, not novelty of content. We attempted to capture the most recent data in our search design additionally incorporating a component of time by the delayed search repetition. Another limitation is that our validation tool does not provide demographics of its workers so this approach replicates the typical Web surfer but not necessarily the typical parent. However, the similarity between the worker and clinical search suggests this was an appropriate proxy.

\section{Awareness of the material childbearing women are downloading in}

\section{preparation for their first birth experience should lead the clinician}

to address potentially outdated obstetric practices, as well as offer

anticipatory guidance regarding best practices in obstetrics and

newborn care.
Birth plans are commonly described as educational tools for expecting and first-time mothers. The access and interface with birth plans via the Web rather than in traditional childbirth education settings removes this process from an educational environment. As a result, many women may interpret questions on a birth plan template as prompts. This study demonstrates that the majority of online birth plans accessible to childbearing women include questions that may lead the woman to make requests outside of what her cliniccian might recommend. Awareness of the material that childbearing women are downloading in preparation for their first birth experience should lead the clinician to address potentially outdated obstetric practices, as well as offer anticipatory guidance regarding best practices in obstetrics and newborn care. Additionally, many of these plans may be effectively incorporated into perinatal education as helpful templates and guides as women start to develop their preferred birth experience. In light of the ubiquity of the utilization of online resources, our data may reassure perinatal educators that encouragement of such independent exploration can positively inform the process of birth plan cultivation. Early directed education will empower women to make informed decisions and offer a forum to explore acceptable alternatives in preparation of a positive birth experience.

\section{REFERENCES}

American Academy of Pediatrics. (1992). Guidelines for perinatal care (3rd ed.). Washington, DC: Author.

American Academy of Pediatrics. (2009). Policy statementAAP publications retired and reaffirmed. Pediatrics, 124(2). http://dx.doi.org/10.1542/peds.2009-1415

American Academy of Pediatrics. (2012). Technical report: Male circumcision. Pediatrics, 130(3). Retrieved from http://pediatrics.aappublications.org/content/pediatrics/130/3/e756.full.pdf

Aragon, M., Chhoa, E., Dayan, R., Kluftinger, A., Lohn, Z., \& Buhler, K. (2013). Perspectives of expectant women and health care providers on birth plans. Journal of Obstetrics and Gynaecology Canada, 35(11), 979-985. http://dx.doi.org/10.1016/S1701-2163(15) 30785-4

Bailey, J. M., Crane, P., \& Nugent, C. E. (2008). Childbirth education and birth plans. Obstetrics \& Gynecology Clinics of North America, 35(3), 497-509. http://dx.doi. org/10.1016/j.ogc.2008.04.005

Carlton, T., Callister, L. C., \& Stoneman, E. (2005). Decision making in laboring women: Ethical issues for perinatal nurses. The Journal of Perinatal Neonatal Neonatal Nursing, 19(2), 145-154. 
Eysenbach, G., \& Köhler, C. (2002). How do consumers search for and appraise health information on the world wide web? Qualitative study using focus groups, usability tests, and in-depth interviews. BMJ, 324(7337), 573-577. http://dx.doi.org/10.1136/bmj.324.7337.573

Goodwin, D. (2011). Top Google result gets 36.4\% of clicks. Search Engine Watch. Retrieved from http:// searchenginewatch.com/article/2049695/Top-GoogleResult-Gets-36.4-of-Clicks-Study

Jansen, B. J., Spink, A., \& Saracevic, T. (2000). Real life, real users, and real needs: A study and analysis of user queries on the web. Information Processing of Management, 36(2), 207-227. http://dx.doi.org/10. 1016/S0306-4573(99)00056-4

Kaufman, T. (2007). Evolution of the birth plan. Journal of Perinatal Education, 16(3), 47-52. http://dx.doi.org/10. 1624/105812407X217985

Lippi, G., \& Franchini, M. (2011). Vitamin K in neonates: Facts and myths. Blood Transfusion, 9(1), 4-9. http:// dx.doi.org/10.2450/2010.0034-10

Lothian, J. (2006). The good, the bad and the future. JOGNN, 35(2), 295-303.

Mei, J. Y., Afshar, Y., Gregory, K. D., Kilpatrick, S. J., \& Esakoff, T. F. (2016). Birth plans: What matters for birth experience satisfaction. Birth, 43(2), 144-150. http://dx.doi.org/10.1111/birt.12226

Pennell, A., Salo-Coombs, V., Herring, A., Spielman, F., \& Fecho, K. (2011). Anesthesia and analgesia-related preferences and outcomes of women who have birth plans. Journal of Midwifery \& Women's Health, 56(4), 376-381. http://dx.doi.org/10.1111/j.1542-2011.2011.00032.x
Rost, J., Johnsmeyer, B., \& Mooney, A. (2014). Diapers to diplomas: What's on the mind of new parents. Thinkwith Google. Retrieved from https://www.thinkwithgoogle .com/consumer-insights/new-parents/

The American College of Obstetricians and Gynecologists. (2006).ACOG practice bulletin no. 76: Postpartum hemorrhage. Obstetrics \& Gynecology, 108(4), 1039-1047.

Too, S. K. (1996). Do birthplans empower women? A study of their views. Nursing Standard, 10(31), 33-37. http:// dx.doi.org/10.7748/ns1996.04.10.31.33.c6222

Westhoff, G., Cotter, A. M., \& Tolosa, J. E. (2013). Prophylactic oxytocin for the third stage of labour to prevent postpartum haemorrhage. The Cochrane Database of Systematic Reviews, 2013(10).

CHRISTINA D. YARRINGTON is a Maternal Fetal Medicine doctor in Boston who is committed to preserving the birth experience of women with complex medical conditions. She works at Boston Medical Center, the safety net hospital in Boston, MA. KARI RADOFF is a certified nurse midwife who works at Boston Medical Center as well as the East Boston Community Health Center. She is active with medical education and ensures aspiring physicians learn the normal birth process. CHLOE A. ZERA is a maternal fetal medicine physician at Brigham and Women's hospital who specializes in women with diabetes and the obstetric care of women of size. 